\title{
Real-time PCR detection and speciation of Cryptosporidium infection using Scorpion probes
}

\section{Correspondence \\ Eric R. Houpt \\ erh6k@virginia.edu}

Received 12 April 2006

Accepted 6 June 2006
Suzanne E. Stroup, ${ }^{1}$ Shantanu Roy, ${ }^{2}$ John Mchele, ${ }^{3}$ Venance Maro, ${ }^{3}$ Simon Ntabaguzi, ${ }^{3}$ Abdullah Siddique, ${ }^{2}$ Gagandeep Kang, ${ }^{4}$

Richard L. Guerrant, ${ }^{1}$ Beth D. Kirkpatrick, ${ }^{5}$ Ronald Fayer, ${ }^{6}$ Joel Herbein, ${ }^{7}$ Honourine Ward, ${ }^{8}$ Rashidul Haque ${ }^{2}$ and Eric R. Houpt ${ }^{1}$

\author{
${ }^{1}$ Division of Infectious Diseases and International Health, University of Virginia, Charlottesville, \\ VA 22908, USA \\ ${ }^{2}$ Centre for Health and Population Research, International Centre for Diarrhoeal Disease \\ Research, Dhaka-1000, Bangladesh \\ ${ }^{3}$ Department of Medicine, Kilimanjaro Christian Medical Centre, PO Box 2222, Moshi, Tanzania \\ ${ }^{4}$ Department of Gastrointestinal Sciences and Haematology, Christian Medical College and \\ Hospital, Vellore, India \\ ${ }^{5}$ University of Vermont College of Medicine, Burlington, VT 05401, USA \\ 6US Department of Agriculture, Beltsville, MD 20705, USA \\ ${ }^{7}$ Techlab, Inc., Blacksburg, VA 24060, USA \\ ${ }^{8}$ Department of Medicine, Tufts University, Boston, MA 02111, USA
}

\begin{abstract}
At least eight species of Cryptosporidium can cause human infection and disease. A real-time PCR (qPCR) assay based on the 18S rRNA gene and utilizing a Scorpion probe was developed to detect all human-pathogenic Cryptosporidium without the usual need for nested amplification. Sensitivity of detection in stool samples was highest using a glass bead-based DNA extraction method (under $10^{3}$ oocysts per stool sample). The assay was validated against 123 human stool specimens from Bangladesh and Tanzania, exhibited a sensitivity and specificity of $>91 \%$ versus microscopy, and detected an additional eight microscopy-negative infections. Cryptosporidium parvum-specific and Cryptosporidium meleagridis-specific Scorpion qPCR assays that provided $100 \%$ accurate speciation compared with Vspl RFLP analysis and sequencing were developed subsequently. These Scorpion probe qPCR assays are simpler to perform than existing nested PCR and RFLP methods for diagnosis and epidemiological investigation of cryptosporidiosis.
\end{abstract}

\section{INTRODUCTION}

Since first appreciated as a protozoal pathogen in 1976, Cryptosporidium is now established as a major cause of diarrhoea in humans (Hlavsa et al., 2005). Cryptosporidium hominis and Cryptosporidium parvum are the major species that have emerged from human studies. However, the avian species Cryptosporidium meleagridis has been observed frequently, e.g. it was observed at a rate of $12 \%$ in a large study of Peruvian human immunodeficiency virus (HIV)-infected cryptosporidiosis patients (Cama et al., 2003). Several additional zoonotic species have been reported infrequently, including Cryptosporidium felis, Cryptosporidium canis, Cryptosporidium muris, Cryptosporidium suis and a cervine genotype (Cama et al., 2003; Gatei et al., 2003; Ong et al., 2002; Pedraza-Díaz et al., 2001; Xiao et al., 2001). Species data are important to aid outbreak investigation and provide information about
Cryptosporidium transmission cycles, and may prove clinically relevant (Houpt et al., 2005; Hunter et al., 2004; Okhuysen et al., 1999).

We therefore sought to develop a Cryptosporidium assay that would be sensitive, provide species information and be simple to perform. The mainstay diagnostic modalities of microscopy or antigen detection do not discriminate species. Several PCR tests have been reported for Cryptosporidium; however, most require nested amplification if using faecal samples and an additional RFLP analysis step for speciation (Amar et al., 2004; Coupe et al., 2005; Gatei et al., 2003; Higgins et al., 2001). In this work, a real-time PCR (qPCR) assay is described that maintains reasonable sensitivity without nested amplification by targeting a relatively short fragment (108-125 bp) of the multicopy $18 \mathrm{~S}$ rRNA gene. C. parvum- and C. meleagridis-specific qPCR assays were also developed; these assays were able to provide 
accurate species information on clinical samples from Bangladesh and Tanzania.

\section{METHODS}

Parasites and parasite DNA. C. parvum oocysts and C. hominis strain TU502 oocysts (kindly provided by Saul Tzipori, Tufts University School of Veterinary Medicine, Grafton, USA) were stored at $4{ }^{\circ} \mathrm{C}$ in PBS, sedimented and counted in a haemacytometer before spiking into $200 \mathrm{mg}$ aliquots of parasite-free stool. DNA of C. meleagridis, C. felis and C. muris was obtained from stool specimens from patients from Moshi, Tanzania or Vellore, India (species identity was confirmed by sequencing the amplified $18 \mathrm{~S}$ rRNA product).

Human faecal specimens. Stool specimens were obtained from individuals with and without diarrhoea at the International Centre for Diarrhoeal Diseases and Research, Dhaka, Bangladesh $(n=70)$, and the Kilimanjaro Christian Medical Centre, Moshi, Tanzania $(n=53)$. Informed consent was obtained from all participants and the human experimentation guidelines of the US Department of Health and Human Services, the University of Virginia, the Centre for Health and Population Research (Bangladesh) and the Kilimanjaro Christian Medical Centre Research Ethics Committee were followed. Specimens were tested for Cryptosporidium infection by microscopy after modified acid-fast stain. Available specimens with disparate microscopy/PCR results were tested by antigen detection via ELISA (Cryptosporidium II kit; Techlab) according to the manufacturer's instructions.

DNA extraction. All DNA was extracted from experimentally spiked and human faecal samples using the QIAamp DNA Stool Mini kit (Qiagen) according to the manufacturer's instructions, except that the suspension was incubated in the kit's stool lysis buffer at $95^{\circ} \mathrm{C}$ and a 3 min incubation with InhibitEx tablets was performed. On experimentally spiked specimens, four methods of stool preparation prior to Qiagen DNA extraction were tested. For the glass bead method, $200 \mathrm{mg}$ acid-washed glass beads $(0.5 \mathrm{~mm}$; Sigma) was added to $200 \mathrm{mg}$ stool in Qiagen ASL buffer and the samples were vortexed for $2-5 \mathrm{~min}$. For sonication, stool samples underwent two 1 min sonication bursts on ice water. For the freeze-thaw method, stool samples were subjected to one or six freeze-thaw cycles in liquid nitrogen and a $95^{\circ} \mathrm{C}$ water bath. For the proteinase K/SDS method, $200 \mu \mathrm{l}$ of $200 \mu \mathrm{g}$ proteinase $\mathrm{K} \mathrm{ml}^{-1}$ in $0 \cdot 2 \%$ SDS in water was added to the stool sample, which was then incubated at $55^{\circ} \mathrm{C}$ for $1 \mathrm{~h}$. For the Tanzanian clinical samples, stool samples received six freeze-thaw cycles prior to Qiagen DNA extraction. For the Bangladeshi clinical samples, $1 \mathrm{~g}$ stool sample was concentrated by ether-PBS sedimentation followed by sonication and six cycles of freeze-thaw prior to Qiagen DNA extraction.

Oligonucleotides. Sequences of the $18 \mathrm{~S}$ rRNA gene of $C$. hominis (AF093491), C. parvum (AF164102), C. meleagridis (AF112574), C. canis (AB210854), C. felis (AF112575), C. muris (X64343) and C. suis (AF108861) were obtained from the NCBI database and aligned using CLUSTAL X v1.8 (http://www.ebi.ac.uk/clustalw/). Initially, a PCR assay was designed to amplify all human-pathogenic Cryptosporidium species (forward 5'-GGTTGTATTTATTAGATAAAGAAC-3', reverse 5' -AGGCCAATACCCTACCGTCT-3' and internal probe $5^{\prime}$-GTGACATATCATTCAAGTTTCTGAC-3'). A $C$. parvum-specific assay was designed with a C. parvum/C. hominisspecific forward primer (5'-CTCGACTTTATGGAAGGGTTG- $3^{\prime}$ ), a C. parvum-specific reverse primer (5'-CAGAAACTTGAATGATATGTCACATTTAA-3') and a C. parvum/C. meleagridis-specific internal probe $\left(5^{\prime}\right.$-GAACCAATATAATTGGTGACTC-3'). A C. meleagridis-specific assay was designed with a $C$. meleagridis-specific forward primer (5'-GCGAAAAAACCTGACTTAATGGAAA-3'), a $C$. meleagridis/C. canis-specific reverse primer (5'-GAAACTTGAAT-
GATATGTCACATA-3') and a common Cryptosporidium probe (5'GGTTGTATTTATTAGATAAAG- $\left.3^{\prime}\right)$. Primers and probes demonstrated no deleterious secondary structures or significant identity to non-Cryptosporidium sequences in a BLAST search (http:// www.ncbi.nlm.nih.gov/blast/). Three Scorpion Uni-probes (Proligo) were generated: one with the pan-Cryptosporidium assay's reverse primer linked to the probe; one with the C. parvum-specific assay's reverse primer linked to the probe; and one with the C. meleagridisspecific assay's forward primer linked to the probe. Scorpion Uniprobes incorporated a $5^{\prime}$ reporter dye, a stem-loop sequence, a black-hole quencher and a hexethylene glycol reverse-extension blocker. Probe binding to the respective neosynthesized strands is indicated in Fig. 1.

PCR amplification, RFLP and sequencing. Amplification took place in a $25 \mu \mathrm{l}$ volume containing $1 \times$ PCR buffer (Qiagen), $8.0 \mathrm{mM}$ total $\mathrm{MgCl}_{2}$ (including that contained in the PCR buffer), $0.5 \mathrm{mM}$ of each deoxynucleoside triphosphate, $1.25 \mathrm{U}$ Taq polymerase (HotStarTaq; Qiagen), $2.5 \mu \mathrm{g}$ BSA, 7.5 pmol Scorpion probe, $30 \mathrm{pmol}$ primer and $5 \mu \mathrm{l}$ faecally extracted DNA. Amplification was performed on an iCycler (Bio-Rad) under the following cycling conditions: $95{ }^{\circ} \mathrm{C}$ for $15 \mathrm{~min} ; 40$ cycles of $95^{\circ} \mathrm{C}$ for $15 \mathrm{~s}, 51{ }^{\circ} \mathrm{C}$ for $15 \mathrm{~s}$ and $72^{\circ} \mathrm{C}$ for $20 \mathrm{~s}$; and then $75^{\circ} \mathrm{C}$ for $10 \mathrm{~min}$. RFLP analysis was performed by incubating $12 \mathrm{U}$ VspI (Promega) per $10 \mu \mathrm{l} \mathrm{PCR} \mathrm{pro-}$ duct at $37^{\circ} \mathrm{C}$ for $2 \mathrm{~h}$. Digested products were fractionated on $2.0 \%$ agarose gel and visualized by ethidium bromide $\left(2 \mu \mathrm{g} \mathrm{ml}^{-1}\right)$. For sequencing, amplicons were purified using the QIAquick PCR Purification kit (Qiagen) and sequenced with the appropriate primers on an Applied Biosystems 377 Prism DNA Sequencer at the University of Virginia Biomolecular Research Facility.

Statistics. Proportions were compared by Fisher's exact test. All $P$ values were two-tailed.

\section{RESULTS AND DISCUSSION}

\section{Selection of oligonucleotides}

The multicopy $18 \mathrm{~S}$ rRNA gene was chosen for amplification given the desire to optimize detection sensitivity and based on findings by others that this gene could be successfully amplified from all human-pathogenic Cryptosporidium species (Jiang \& Xiao, 2003). 18S rRNA sequences from the eight Cryptosporidium species that have been reported thus far to cause disease in humans were downloaded from GenBank. Primers and probes were designed to detect all Cryptosporidium species that spanned the widely used VspI C. hominis restriction site (Fig. 1, pan-Cryptosporidium assay). A C. hominis-specific Scorpion assay was developed, but exhibited suboptimal performance (see below). Therefore, $C$. parvum- and C. meleagridis-specific reactions were designed that maximized primer and probe polymorphisms against the other species (Fig. 1, right-hand column).

\section{Sensitivity of the Cryptosporidium Scorpion probe qPCR assays}

The sensitivity of the pan-Cryptosporidium assay was determined on parasite-free stool spiked with known quantities of purified C. parvum or C. hominis oocysts. Four methods of initial stool preparation were tested including glass bead homogenization, sonication, freezethaw cycles and treatment with proteinase $\mathrm{K}$ with SDS. DNA 


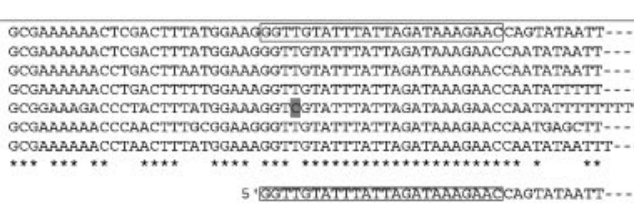

$18 S$ rRNA sequence

\section{C. parvum-specific assay} species

18S rRNA sequence

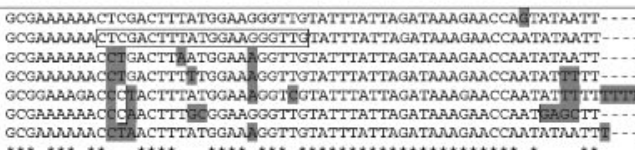
(of primers/probe vs. sequence)

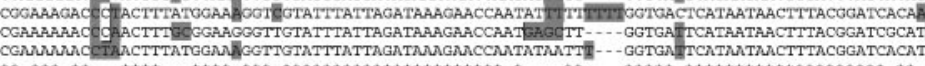

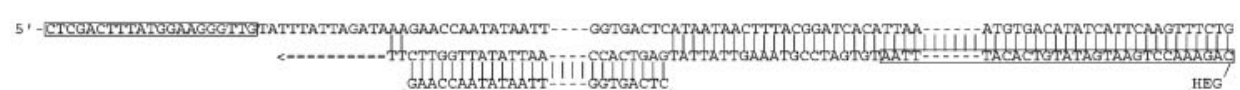

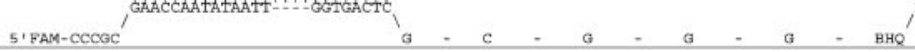

C. meleagrids-specific assay species

c. hominis
c. parvum
c. meleagridis
c. canis
c. felis
c. muris
c. suis

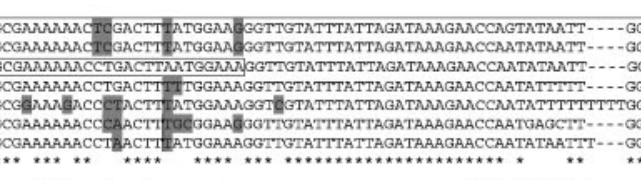

18S rRNA sequence

c. meleagridis Вне - $-G-G-C-\sigma$

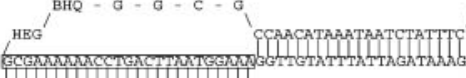

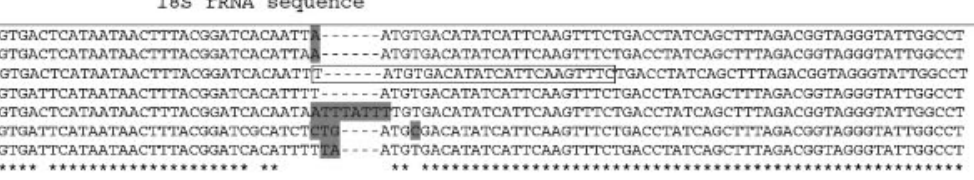
\# mismatches
(of primers/probe vs. sequence)
5 1.

Fig. 1. Primer and Scorpion probe sequences for pan-Cryptosporidium, C. parvum-specific and C. meleagridis-specific qPCR. 18S rRNA sequences for C. hominis, C. parvum, C. meleagridis, C. canis, C. felis, C. muris and C. suis were obtained from GenBank (nt 172-322 of AF093491, AF164102, AF112574 and AB210854, nt 173-333 of AF112575, nt 150-302 of X64343 and nt 172-325 of AF108861 are shown for the seven Cryptosporidium species, respectively) and aligned using CLUSTAL software. Primers (boxed) and probes (bold) were designed to detect all Cryptosporidium species, C. parvum or C. meleagridis. Sequence mismatches of primers or probes against other species are highlighted and shown in the column on the right. Probes were linked to forward or reverse primers via Scorpion Uni-probes as indicated. Separation of fluorophore (HEX, FAM or TEX) from the black-hole quencher (BHQ) during PCR synthesis is shown below sequences. HEG, Hexethylene glycol reverseextension blocker. The $V s p l$ restriction site, AT $\downarrow$ TAAT (underlined in the uppermost $C$. hominis sequence), is exclusive to C. hominis. 
(a)

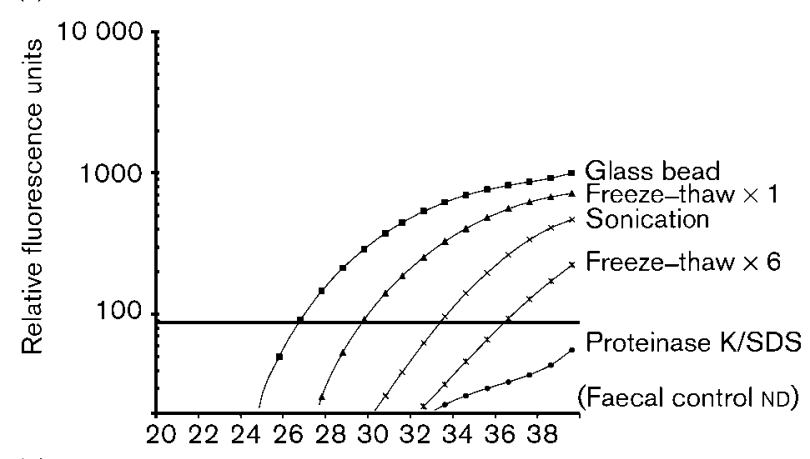

(b)

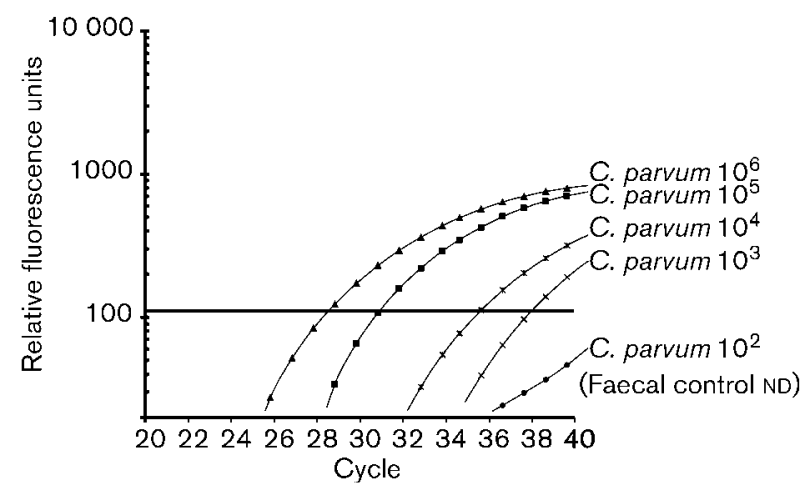

Fig. 2. Sensitivity of the Scorpion probe pan-Cryptosporidium qPCR assay. (a) $10^{5}$ C. parvum oocysts were spiked into aliquots of parasite-free stool, which were then homogenized via glass bead, freeze-thaw, sonication or proteinase K/SDS methods. DNA was extracted with the QIAamp DNA Stool Mini kit. Samples were then amplified with the pan-Cryptosporidium Scorpion qPCR assay. (b) Serial dilutions of C. parvum oocysts were spiked into parasite-free stool. DNA was prepared via the glass bead/Qiagen method and qPCR-amplified with the panCryptosporidium assay. No fluorescence was observed with unspiked faecal samples or water controls (data not shown). The fluorescence threshold for each run was calculated by BioRad iCycler software v3.0 and is shown as a horizontal line. ND, Not detected.

was then purified from all preparations using the commercial QIAamp DNA Stool Mini kit and subjected to qPCR. The lowest cycle threshold $\left(\mathrm{C}_{\mathrm{T}}\right)$ was consistently observed on the glass bead-prepared samples or after one cycle of freeze-thaw (Fig. 2a). The lower limit of detection with this assay was $10^{2}-10^{3}$ oocysts per $200 \mathrm{mg}$ stool sample after 40 cycles of PCR (Fig. 2b). Sensitivity of the C. parvum- and $C$. meleagridis-specific Scorpion assays for detecting C. parvum and $C$. meleagridis, respectively, was similar to that of the pan-Cryptosporidium assay (data not shown).

\section{Specificity of the Cryptosporidium Scorpion probe $q P C R$ assays}

Specificity of the assays was examined. For C. hominis and $C$. parvum, experimentally spiked specimens were used, but sequence-confirmed faecal specimens were used for $C$. meleagridis, C. felis and C. muris. The pan-Cryptosporidium assay detected all samples (Fig. 3a), including C. felis and $C$. muris, despite their $1 \mathrm{bp}$ sequence mismatches in the forward primer and probe, respectively (Fig. 1). In contrast, the C. parvum-specific assay detected only C. parvum (Fig. 3b) and the C. meleagridis-specific assay detected only C. meleagridis (Fig. 3c). Several C. hominis-specific Scorpion probes were attempted within this region; however, all exhibited poor sensitivity or false-positive detection of $C$. parvum (data not shown). Such difficulty has been reported previously due to the minimal nucleotide polymorphisms in the $18 \mathrm{~S}$ rRNA sequence of Cryptosporidium species (Higgins et al., 2001). Finally, multiplex PCR with all primers and probes could be performed, but this is not recommended due to diminished sensitivity versus singleplex reactions (e.g. C. hominis $\mathrm{C}_{\mathrm{T}}$ changed from $32 \cdot 8$ to $35 \cdot 1$ and $C$. meleagridis $\mathrm{C}_{\mathrm{T}}$ changed from $29 \cdot 1$ to $33 \cdot 1$ ).

\section{Comparison of Cryptosporidium Scorpion probe qPCR with microscopy on clinical specimens}

The pan-Cryptosporidium assay was validated on 123 banked stool DNA samples obtained during case-control studies in Bangladesh $(n=70)$ and Tanzania $(n=53)$ during the preceding 3 years. Sixty-three patients had diarrhoea $(\geqslant 3$ loose or watery stools over the previous $24 \mathrm{~h}$ ), 60 did not and all 53 Tanzanian patients were HIV-infected. Fresh specimens were tested by microscopy after acid-fast staining and then stored at $-70{ }^{\circ} \mathrm{C}$. DNA was then extracted via a freeze-thaw/Qiagen method (note that sufficient quantities were not available for re-extraction via the more sensitive glass bead method). The qPCR assay exhibited a sensitivity of $92 \%$ (95\% confidence interval of 78-98\%) and a specificity of $91 \%$ (95\% confidence interval of $83-96 \%$ ) versus microscopy. Specifically, of 36 microscopy-positive specimens, 33 were qPCR-positive; of 87 microscopynegative specimens, 79 were qPCR-negative. For specimens with inconsistent qPCR/microscopy results, available samples were retested by Cryptosporidium ELISA; results showed that all three microscopy-positive/qPCR-negative specimens were antigen-negative and six of the eight microscopynegative/qPCR-positive specimens were antigen-positive. Overall, therefore, the qPCR sensitivity appeared to exceed that of microscopic analysis and that reported for traditional PCR assays for Cryptosporidium (Kostrzynska et al., 1999), perhaps due to the short ( $\sim 125 \mathrm{bp})$ target sequence, which increases PCR efficiency. Sensitivity of PCR in faecal specimens has historically been a limitation for Cryptosporidium such that nested amplification has frequently been used (Bialek et al., 2002; Morgan et al., 1998). Whereas the sensitivity of the present assay would be predicted to improve with nested amplification, we do not recommend this because of the risks of contamination and increased reagent cost of nested PCR, but rather favour future use of the glass bead method for DNA extraction (Fig. 2). 

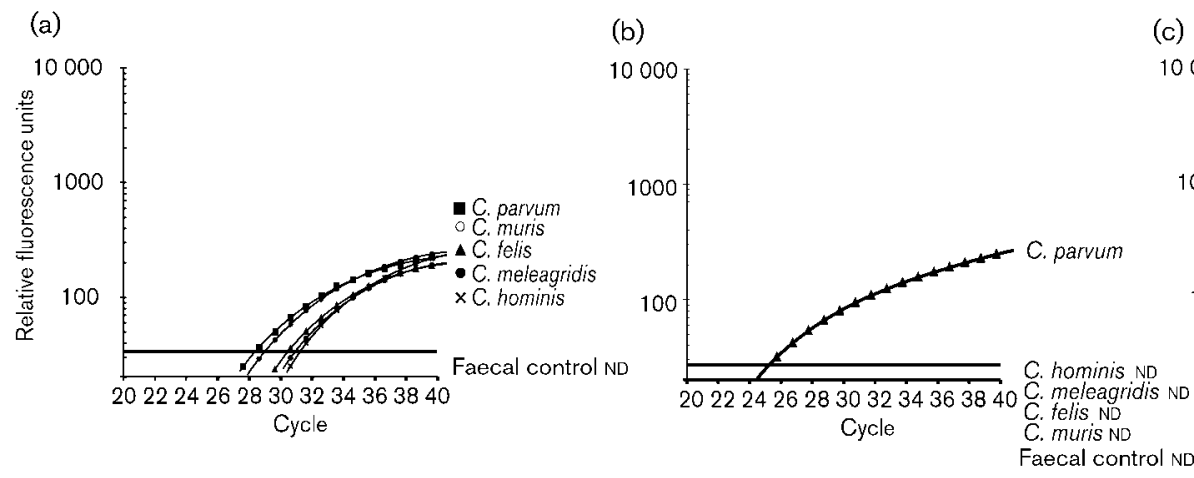

(c)

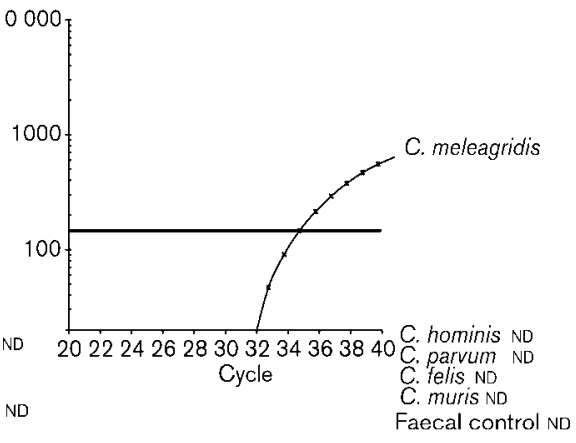

Fig. 3. Specificity of the C. parvum- and C. meleagridis-specific Scorpion probe qPCR assays. DNA was extracted from parasite-free stool spiked with $10^{6} \mathrm{C}$. hominis or $10^{6} \mathrm{C}$. parvum oocysts, as well as from human faecal specimens infected with C. meleagridis, C. felis and C. muris (confirmed by sequencing of the $18 \mathrm{~S}$ rRNA gene). DNA samples were qPCRamplified using (a) the pan-Cryptosporidium assay, (b) the C. parvum-specific assay and (c) the C. meleagridis-specific assay. The fluorescence threshold for each run was calculated by Bio-Rad iCycler software v3.0 and is shown as a horizontal line. ND, Not detected.

\section{Speciation using Scorpion probes}

The accuracy of the C. parvum and C. meleagridis assays for species identification compared with VspI RFLP digestion analysis was examined. First, each of the 82 panCryptosporidium qPCR-negative specimens was tested by the C. parvum- and C. meleagridis-specific qPCR assays and no false positives were observed (Table 1). Upon testing each of the 41 pan-Cryptosporidium qPCR-positive specimens with the specific qPCR assays, 26 specimens were $C$. parvum qPCR-negative/C. meleagridis qPCR-negative, 12 were C. parvum qPCR-positive/C. meleagridis qPCRnegative, two were $C$. parvum qPCR-positive/C. meleagridis qPCR-positive and one was $C$. parvum qPCR-negative/ $C$. meleagridis qPCR-positive. Each amplicon from the panCryptosporidium qPCR, C. parvum-specific qPCR and $C$. meleagridis-specific qPCR assays (total $=58$ ) was then analysed by VspI RFLP digestion to confirm species identity and identify $C$. hominis infections (the only species with the internal VspI site; Fig. 1). This analysis revealed no VspI digestion of any C. parvum-specific or C. meleagridisspecific qPCR product. Furthermore, for the two C. parvum
qPCR-positive/C. meleagridis qPCR-positive specimens, both the C. parvum $\mathrm{qPCR}$ and $C$. meleagridis $\mathrm{qPCR}$ products were sequenced and the presence of both species was confirmed. Finally, two of the 26 pan-Cryptosporidium qPCR-positive/C. parvum qPCR-negative/C. meleagridis qPCR-negative specimens that were not digested with $V s p$ I were sequenced and were found to be $C$. felis infections (the first $C$. felis infections reported from Bangladesh). Overall, therefore, the C. parvum- and C. meleagridisspecific qPCR assays were $100 \%$ accurate compared with $V s p$ I RFLP and sequencing analysis. By combining panCryptosporidium and C. parvum-specific results, C. hominis infection could be reasonably inferred from a panCryptosporidium qPCR-positive/C. parvum qPCR-negative result (24 of 27 such samples were confirmed as $C$. hominis by RFLP analysis).

Interestingly, six of 14 C. parvum infections were mixed infections compared with only four of $28 \mathrm{C}$. hominis infections $(P=0 \cdot 06)$. Mixed $C$. hominis/C. parvum infections have been clearly documented in up to $4-12 \%$ of cases in diverse regions including the United States, Uganda and

Table 1. Speciation using C. parvum- and C. meleagridis-specific qPCR assays

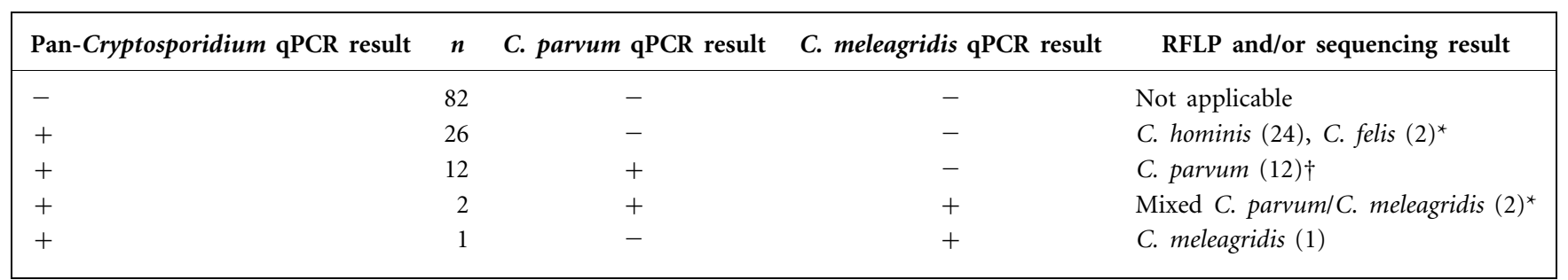

${ }^{*}$ The presence of $C$. felis and mixed C. parvum/C. meleagridis infection was confirmed by sequencing.

$\dagger$ Four of these 12 C. parvum infections were found to be mixed C. parvum/C. hominis infections, as shown by VspI digestion of the panCryptosporidium qPCR amplicon without VspI digestion of the C. parvum-specific qPCR amplicon. 
Peru (Cama et al., 2003; Tumwine et al., 2005). Some have reported that this burden may be underestimated since standard PCR-RFLP assays for Cryptosporidium are not very sensitive for detection of the minor subtype (Reed et al., 2002). The significance of human infection with particular Cryptosporidium species, mixed or not, remains to be elucidated on many levels. It is hoped that these assays can serve as a tool towards that end.

\section{ACKNOWLEDGEMENTS}

This work was supported by Public Health Service grant AI056872-02 and AI043596-06 from the National Institute of Allergy and Infectious Diseases, National Institutes of Health. We thank William A. Petri, Jr, for helpful discussions.

\section{REFERENCES}

Amar, C. F., Dear, P. H. \& McLauchlin, J. (2004). Detection and identification by real time PCR/RFLP analyses of Cryptosporidium species from human faeces. Lett Appl Microbiol 38, 217-222.

Bialek, R., Binder, N., Dietz, K., Joachim, A., Knobloch, J. \& Zelck, U. E. (2002). Comparison of fluorescence, antigen and PCR assays to detect Cryptosporidium parvum in fecal specimens. Diagn Microbiol Infect Dis 43, 283-288.

Cama, V. A., Bern, C., Sulaiman, I. M. \& 7 other authors (2003). Cryptosporidium species and genotypes in HIV-positive patients in Lima, Peru. J Eukaryot Microbiol 50 (Suppl.), 531-533.

Coupe, S., Sarfati, C., Hamane, S. \& Derouin, F. (2005). Detection of Cryptosporidium and identification to the species level by nested PCR and restriction fragment length polymorphism. J Clin Microbiol 43, 1017-1023.

Gatei, W., Greensill, J., Ashford, R. W., Cuevas, L. E., Parry, C. M., Cunliffe, N. A., Beeching, N. J. \& Hart, C. A. (2003). Molecular analysis of the $18 \mathrm{~S}$ rRNA gene of Cryptosporidium parasites from patients with or without human immunodeficiency virus infections living in Kenya, Malawi, Brazil, the United Kingdom, and Vietnam. $J$ Clin Microbiol 41, 1458-1462.

Higgins, J. A., Fayer, R., Trout, J. M., Xiao, L., Lal, A. A., Kerby, S. \& Jenkins, M. C. (2001). Real-time PCR for the detection of Cryptosporidium parvum. J Microbiol Methods 47, 323-337.

Hlavsa, M. C., Watson, J. C. \& Beach, M. J. (2005). Cryptosporidiosis surveillance - United States 1999-2002. MMWR Surveill Summ 54, $1-8$.
Houpt, E. R., Bushen, O. Y., Sam, N. E. \& 8 other authors (2005). Short report: asymptomatic Cryptosporidium hominis infection among human immunodeficiency virus-infected patients in Tanzania. Am J Trop Med Hyg 73, 520-522.

Hunter, P. R., Hughes, S., Woodhouse, S., Raj, N., Syed, Q., Chalmers, R. M., Verlander, N. Q. \& Goodacre, J. (2004). Health sequelae of human cryptosporidiosis in immunocompetent patients. Clin Infect Dis 39, 504-510.

Jiang, J. \& Xiao, L. (2003). An evaluation of molecular diagnostic tools for the detection and differentiation of human-pathogenic Cryptosporidium spp. J Eukaryot Microbiol 50 (Suppl.), 542-547.

Kostrzynska, M., Sankey, M., Haack, E. \& 8 other authors (1999). Three sample preparation protocols for polymerase chain reaction based detection of Cryptosporidium parvum in environmental samples. J Microbiol Methods 35, 65-71.

Morgan, U. M., Pallant, L., Dwyer, B. W., Forbes, D. A., Rich, G. \& Thompson, R. C. (1998). Comparison of PCR and microscopy for detection of Cryptosporidium parvum in human fecal specimens: clinical trial. J Clin Microbiol 36, 995-998.

Okhuysen, P. C., Chappell, C. L., Crabb, J. H., Sterling, C. R. \& DuPont, H. L. (1999). Virulence of three distinct Cryptosporidium parvum isolates for healthy adults. J Infect Dis 180, 1275-1281.

Ong, C. S., Eisler, D. L., Alikhani, A., Fung, V. W., Tomblin, J., Bowie, W. R. \& Isaac-Renton, J. L. (2002). Novel Cryptosporidium genotypes in sporadic cryptosporidiosis cases: first report of human infections with a cervine genotype. Emerg Infect Dis 8, 263-268.

Pedraza-Díaz, S., Amar, C., Iversen, A. M., Stanley, P. J. \& McLauchlin, J. (2001). Unusual Cryptosporidium species recovered from human faeces: first description of Cryptosporidium felis and Cryptosporidium 'dog type' from patients in England. $J$ Med Microbiol 50, 293-296.

Reed, C., Sturbaum, G. D., Hoover, P. J. \& Sterling, C. R. (2002). Cryptosporidium parvum mixed genotypes detected by PCR-restriction fragment length polymorphism analysis. Appl Environ Microbiol 68, 427-429.

Tumwine, J. K., Kekitiinwa, A., Bakeera-Kitaka, S., Ndeezi, G., Downing, R., Feng, X., Akiyoshi, D. E. \& Tzipori, S. (2005). Cryptosporidiosis and microsporidiosis in Ugandan children with persistent diarrhea with and without concurrent infection with the human immunodeficiency virus. Am J Trop Med Hyg 73, 921-925.

Xiao, L., Bern, C., Limor, J., Sulaiman, I., Roberts, J., Checkley, W., Cabrera, L., Gilman, R. H. \& Lal, A. A. (2001). Identification of 5 types of Cryptosporidium parasites in children in Lima, Peru. J Infect Dis 183, 492-497. 